\title{
A DISTANCE SCALE FROM THE IR MAGNITUDE/HI VELOCITY WIDTH RELATION
}

\author{
M. Aaronson \\ Steward Observatory \\ University of Arizona \\ Tucson, Arizona 85721 \\ U.S.A.
}

\begin{abstract}
A summary is given of recent work using the IR/H I method, arguably the best global distance indicator presently available. Reflex motion toward the microwave dipole anisotropy has been seen relative to a sample of nearby galaxy clusters; this result is contrasted with the somewhat divergent conclusions obtained by Burstein et al. from a similar study of elliptical galaxies. A best guess calibration of the IR/H I zero point continues to lead to a high value for the expansion rate. This finding cannot be explained by appealing to Malmquist bias, as demonstrated by a straightforward linearity test of the velocity-distance relation. However, all current estimates of the Hubble constant are plagued by the large uncertainties to the distances of nearby calibrating galaxies, a problem whose full solution probably must await the launch of the Hubble Space Telescope.
\end{abstract}

\section{INTRODUCTION -- ARE ANY GALAXY DISTANCES SECURE?}

It is widely acknowledged that current estimates of the Hubble constant are uncertain by a factor perhaps as large as two. The underlying reasons for this situation are usually obscured by claims and counterclaims involving magnitude bias effects on samples of distant galaxies. It is the author's belief, however, that the principal problem in all current determinations of $\mathrm{H}_{0}$ starts right in our own backyard with the rubbery state of the nearby distance scale. I would like to begin this contribution by illustrating the point with three cases: the Galaxy, the LMC, and M33.

Turning first to the Milky Way, the last few years has seen a dramatic reduction in measures of the galactic center distance $R_{0}$. As a result, the old recommended IAU value of $10 \mathrm{kpc}$ was changed at the 1985 General Assembly to $8.5 \mathrm{kpc}$ (Kerr and Lynden-Bell 1986). Even so, the best recent determinations of $R_{0}$ from four independent methods indicate a smaller value still. From $R R$ Lyraes, Blanco and Blanco (1985) obtain $R_{0}=7.3+0.5 \mathrm{kpc}$. Using globulars, Frenk and. White (1982) have found $R_{0}{ }^{-}=6.8+0.8 \mathrm{kpc}$. Reid et al. (1987) have 
measured $R_{0}=7+1 \mathrm{kpc}$ from maser expansion velocities. Finally, kinematic modeling of field Cepheids calibrated via main sequence fitting leads Caldwell and Coulson (1987) to propose $R_{0}=7.8+0.7$ kpc. The straight mean of these four results is $\sim 30 \%$ less than the ( not so) old IAU value?

Well, perhaps we should not be too surprised that it is difficult to obtain an accurate distance to a place hidden by 30 magnitudes of visual extinction, so let us consider instead the Large Cloud, our next nearest companion galaxy. In one way or another, modern distance estimates to most other galaxies scale directly with the LMC modulus, giving it a high significance. This fact should be born in mind by the reader when confronted with what is often the "blind" averaging of discrepant distances to farther systems. The most oft-quoted LMC modulus is based on Cepheid work of the South African group. Martin, Warren, and Feast (1979) gave $18.69 \pm 0.15$, based on a zero-point calibration again obtained from galactic main sequence fitting. Little changed between this and the more recent Cepheid study of Caldwell and Coulson (1986), where a value of $18.65+0.07$ was offered.

For the last few years, however, there has been continually mounting evidence that the Cepheid distance was problematic, first coming from the RR Lyraes (see the discussion following Graham 1984), then from the reduced galactic cluster distances found using Stromgren photometry by Schmidt (1984) and subsequently by Balona and Shobbrook (1984), from differences in the Mira P-L relations (see Menzies and Whitelock 1985), and from direct main sequence fitting to the LMC itself (Schommer, Olszewski, and Aaronson 1984). A shorter LMC modulus had of course been advocated for a long time by Eggen (1977, see also de Vaucouleurs 1980).

Recently, the South African work seems to have come into better agreement, with the latest result being a modulus of $18.45+0.05$ (Caldwell and Coulson 1987), a $3 \sigma$ change from their earlier effort! The difference is largely due to the number of very fine CCD color-magnitude diagrams for galactic calibrating clusters that have emerged in the last few years which have tended to yield systematically smaller moduli by $\sim 0.2 \mathrm{mag}$ (in accord with the Stromgren findings). For comparison, recent $R R$ Lyrae studies by Walker (1985) and Reid and Strugnell (1986) give LMC moduli of $18.42+0.10$ and $18.37+0.15$, respectively. The luminosity calibration of $O B$ stars lead Conti, Garmany, and Massey (1986) to propose $18.3+0.3$. Within the errors, all these values are in agreement with $18.2+\overline{0} .2$ obtained by Schommer et al. (1984) via Cloud main sequeñce fitting. (Convective overshooting does not alter this last result, since fits are made to the unevolved main sequence. As discussed by Schommer 1986, the luminosity function method advocated by Chiosi and Pigatto 1986 cannot independently determine age and distance, and is itself subject to the uncertainties of convective effects, so is therefore not to be preferred.) Finally, we note that application of stellar evolutionary models to the clump luminosities of Cloud clusters was found by Seidel, Da Costa, and Demarque (1986) to yield consistent results only for the "short scale." 
The dust has by no means settled on the LMC distance, though a best guess modulus would seem to fall in the range $18.3-18.4$. Once again, within a few years time, a "firm" distance has been modified by $\sim 15-20 \%$. It should be remembered that this has in part come about because the data base of material for the Magellanic Clouds is far more extensive than for any other extragalactic systems.

Finally, we turn to M33. Two recent studies involving Cepheids, one based on photographic photometry by Sandage and Carlson (1983), and another on multi-color CCD observations by Freedman (1985), yield moduli of 25.35 and 24.1 , respectively, a difference of 1.25 magnitudes! Although the former value is an apparent modulus, it is nonetheless roughly the same one used by Sandage and Tammann (1984) in their attempt to derive a Hubble constant from the IR/H I relation. (These authors also adopt a large M81 apparent modulus of 28.8, which Sandage 1986 has indicated to be based primarily on novae. Use of the latter as reliable distance indicators, though, has been called into question by the recent work of Pritchet and van den Bergh 1986 on novae in the Virgo cluster.)

It is illuminating to examine the origin of this gross difference in M33 modulus. Reddening is part of the problem; Freedman's multicolor data convincingly demonstrates the presence of a primarily internal extinction of $\mathrm{AV}_{\mathrm{V}} \sim 0.6 \mathrm{mag}$. A difference in Cepheid calibrating precepts also seems involved. The relative LMC-M33 distance quoted by Sandage and Carlson (1983) implies an LMC modulus of 19.0, whereas Freedman (1985) adopts 18.5 mag. This leaves a scale difference of only $\sim 0.2 \mathrm{mag}$, which presumably rests almost entirely with the less precise photographic work. (A larger scale error appears to be present for Hubble's Cepheids as corrected by Sandage and Carlson; in this regard, a similar conclusion has been reached by Christian and Schommer 1986.)

Well, what is one to make of the above. First of all, the answer to the question posed in the section heading should be obvious. Secondly, it is also clear that all of the older photographic work is in desperate need of checking via multi-wavelength CCD observations and modern point-spread-function fitting reduction techniques. Furthermore, it is interesting to note that the distance scale within the Local Group appears to be undergoing a considerable shrinkage, which will certainly have important implications with regard to future efforts to measure $\mathrm{H}_{0}$.

\section{DEVIATIONS IN THE HUBBLE FLOW}

Departures from uniformity in the Hubble flow can provide important information about the value of $\Omega$ and the processes involved in galaxy formation. For a number of years, the author and collaborators have been involved in the study of large scale flow deviations using the IR/H I relation. This method satisfies most of the desirable qualities one would like to have in a distance indicator based on the global properties of galaxies: The technique connects with physics via Newton's law of gravity, all of the measurables involved are 
quantitative, extinction effects are not problematic, the method can be employed over a wide distance range, and the scatter in the relation is demonstrably small (e.g. Aaronson and Mould 1986).

Our initial interest in mapping the Hubble flow lay with determining the Local Group infall velocity toward Virgo. For this purpose a flow-model analysis was made of $\sim 300$ galaxies within the Local Supercluster (Aaronson et al. 1982, hereafter AHMST). An infall in the range $250-300 \mathrm{~km} \mathrm{~s}^{-1}$ was found, along with a significant random component of Local Group motion. More recently, Lilje, Yahil, and Jones (1986) have reanalyzed the AHMST data set by also allowing for the presence of a quadrupolar tidal velocity field. Most of the peculiar motion seen by AHMST can be accounted for with the presence of such a tidal field. It is interesting that while Lilje et al. argue the form of the tidal field indicates the existence of more than a single attractor, they find the expansion axis to point in the general direction of the Hydra-Centaurus Supercluster, and further suggest the size of the field implies a perturber distance roughly three times greater than the Virgo cluster. We come back to these points below.

Our most recent work with the IR/H I relation has involved the study of ten nearby galaxy clusters in the redshift range 4,000 $11,000 \mathrm{~km} \mathrm{~s}^{-1}$ (Aaronson et al. 1986, hereafter ABMHSC). Two results on large scale velocities have emerged from this effort. First, a Virgocentric motion of $\sim 300 \mathrm{~km} \mathrm{~s}^{-1}$ is indicated, in good agreement with the earlier analysis of the Local Supercluster sample. Second, the reflex motion of the Local Group relative to the cluster sample yields a vector that agrees well in both magnitude and direction with the velocity inferred from the dipole anisotropy of the microwave background.

The difference between the dipole vector and Local Group motion within the Local Supercluster yields an estimate of bulk Supercluster motion as a whole. The direction of this bulk motion is again found to be toward Hydra-Centaurus; its magnitude, however, is not so well determined, and can be as low as 300 or as high as $500 \mathrm{~km} \mathrm{~s} \mathrm{~s}^{-1}$, depending on precisely what is assumed for the tidal/peculiar motion of the Local Group (see ABMHSC, Lilje et al. 1986). In any event, these results and those of the tidal field work point toward Virgo and Hydra-Centaurus as the two principle attractors giving rise to the dipole anisotropy.

A further result to follow from the ABMHSC study concerns relative cluster-cluster motions. After correcting the velocities for the dipole effect, the observed scatter of the ten cluster sample about purely uniform Hubble flow is only $\sim 270 \mathrm{~km} \mathrm{~s}^{-1}$. An amount larger than this would be expected from the formal errors in velocity and distance alone. The latter, though, were overestimated since they did not make any allowance for subclustering. If zero error is assumed in the observables, then it would seem that $270 \mathrm{~km} \mathrm{~s}^{-1}$ was a firm upper limit to the 1-d bulk motion of a typical cluster, or $470 \mathrm{~km} \mathrm{~s}^{-1}$ for the $3-\mathrm{d}$ bulk motion, comparable with our measure for the Local Supercluster.

The above findings stand in somewhat sharp contrast to those recently reported by Burstein et al. (1986, hereafter BDDFLTW), who have applied an improved version of the Faber-Jackson relation to an 
all-sky sample of elliptical galaxies. The resulting reflex vector (V $=470 \mathrm{~km} \mathrm{~s} \mathrm{~s}^{-1}$ toward $\ell=183^{\circ}, \mathrm{b}=29^{\circ}$ ) points in an orthogonal direction to the dipole velocity $\left(\mathrm{V}=600 \mathrm{~km} \mathrm{~s}-1\right.$ to $\left.\ell=268^{\circ}, \mathrm{b}=27^{\circ}\right)$, such that when these two components are subtracted from one another, a residual bulk motion of $600 \mathrm{~km} \mathrm{~s}^{-1}$ still remains, pointing once again roughly towards Hydra-Centaurus. However, the bulk motion now involves a scale that extends outwards from the Local Group some $4000 \mathrm{~km} \mathrm{~s}{ }^{-1}$, actually encompassing Hydra-Centaurus itself. That is, the latter is claimed to be moving with a velocity comparable to the Local Group and in the same direction, so that the attractor must lie well beyond. (A similar, though apparently less statistically significant result, has also been reported by Collins, Joseph, and Robertson 1986.)

Although the conclusions of ABMHSC and BDDFLTW look very different, the results are not formally inconsistent, as the two data sets overlap very little spatially or in redshift. In fact, for the few clusters in common between the two samples, the agreement in relative modulus appears to be excellent. Most of the power in the BDDFLTW solution comes from $E$ galaxies in the redshift range 2000 $4000 \mathrm{~km} \mathrm{~s}^{-1}$, whereas the cluster sample of ABMHSC does not start until $4000 \mathrm{~km} \mathrm{~s}^{-1}$. The latter is confined to a broad ring around the sky defined by the Arecibo declination range, and is least sensitive to the direction of the BDDFLTW motion, which is roughly perpendicular to the Arecibo band. Nevertheless, the implication is that on average most structures beyond $\sim 4000 \mathrm{~km} \mathrm{~s}^{-1}$ would be co-moving with respect to the microwave frame.

The BDDFLTW results do appear to differ with those of AHMST. The former authors claim that their $0-2000 \mathrm{~km} \mathrm{~s}^{-1}$ shell yields the same reflex vector as the whole sample, i.e., they find no signal indicating strong Virgocentric motion. However, they have not fit a proper flow model to the data; because of the way the velocity vectors then cancel, this will lead to a result biased toward small infall values (Yahil 1986). Furthermore, while the $600 \mathrm{~km} \mathrm{~s}^{-1}$ bulk velocity found by BDDFLTW is perhaps just marginally consistent with the typical $1-\mathrm{d}$ bulk motions indicated by the ABMHSC study, it is definitely a problem for theoretical models of biased galaxy formation with cold dark matter (e.g. Vittorio, Juszkiewicz, and Davis 1986). Such models imply that large scale bulk velocities should not be greater than $\sim 150 \mathrm{~km} \mathrm{~s} \mathrm{~s}^{-1}$. There have been suggestions that the BDDFLTW result and the earlier Rubin-Ford effect could be biased by spatial inhomogeneities in the samples (see Vittorio et al. 1986), and it is interesting to note in this regard the recent study of the Centaurus cluster by Lucey, Currie, and Dickens (1986). These authors find Centaurus to be composed of two distinct clumps separated in redshift by $\sim 1500 \mathrm{~km} \mathrm{~s}^{-1}$. They argue, though, that the clumps lie at the same distance, and are just now merging. The cosmological implications of such large scale velocity flows are clearly very substantial, and the whole problem is obviously deserving of much greater study. 


\section{A HUBBLE CONSTANT FROM THE IR/H I METHOD}

As discussed in the first section, the greatest difficulty in trying to derive a value for $\mathrm{H}_{0}$ using the $\mathrm{IR} / \mathrm{H}$ I relation (or any other global distance indicator) arises from knowing what moduli to select for nearby calibrating galaxies. A judicious choice of moduli can lead to an expansion rate that is near just about anyone's preferred value. Nevertheless, the author believes it is possible to lay down a sensible set of ground rules that should be followed in treating the problem.

The first of these rules is to work with galaxy clusters. This avoids the thorny issue of how to properly treat the Malmquist effect, because the galaxies in a cluster are generally all at the same distance. In fact, because the cluster objects are ultimately $H$ I selected, it is likely that any sort of magnitude bias effects can be dispensed with entirely. Support for this contention can be seen in Figure 3 of Aaronson and Mould (1986), which shows no significant departure from a linear velocity-distance relation over a factor of eight in radial velocity. (The test is precisely that advocated by Tammann at this Symposium to check for Malmquist bias.) We also note in this regard the excellent agreement in relative Virgo-Coma modulus measured by ABMHSC from the $I R / H$ I relation $[\Delta(\mathrm{m}-\mathrm{M})=3.69 \mathrm{mag}]$ and that obtained by BDDFLTW from their improved Faber-Jackson method $[\Delta(\mathrm{m}-\mathrm{M})=3.65 \mathrm{mag}]$. Sandage and Tammann (1984) themselves apparently accept this latter approach as bias free.

In choosing a proper set of calibrating objects, one should also place restrictions on inclination (we have followed $i>45^{\circ}$, which may be too optimistic), morphology (types no earlier than Sab or later than $\mathrm{Sdm})$, and corrected velocity width $\left(200<\Delta \mathrm{V}<600 \mathrm{~km} \mathrm{~s}^{-1}\right.$ probably being a good choice). The last of these criteria is driven by the desirability both to cover the same range in width sampled by the more distant cluster spirals, and to avoid the regime of small linewidth objects where the IR/H I relation is not well defined. The relation may in fact break down entirely for dwarfish galaxies, since inclination as judged from the optical structure often bears little resemblance to that indicated from detailed H I maps (Bothun 1986).

Finally, the calibrators should be confined to those systems having modern multi-color CCD (or good IR) Cepheid photometry, with no assumptions about group membership being introduced. This last criteria is particularly restrictive, and reduces the number of acceptable objects to just three: M31, M33, and NGC 2403. As discussed by ABMHSC, these galaxies yield a high value for the expansion rate, $\mathrm{H}_{0} \sim 90 \mathrm{~km} \mathrm{~s}^{-1} \mathrm{Mpc}^{-1}$.

Tammann at this Symposium (see also Kraan-Korteweg, Cameron, and Tammann 1986) has attempted to analyze the biases in the nearby field sample of AHMST, claiming that a smaller value of $\mathrm{H}_{0}$ thereby follows. There are three possible ways to reconcile his results with the above: either (1) cluster and field spirals differ in their IR/H I properties; or (2) a different set of calibrating distances and/or calibrating precepts has been employed (involving, for instance, the inappropriate extrapolation of the quadratic form of the relation down to the low-luminosity regime, as was indicated in the oral version of 
Tammann's talk); or (3) an incorrect analysis of bias in the field sample. There is considerable evidence to suggest that the first of these possibilites has no basis. In particular, the clusters studied by ABMHSC cover a wide range of type, from dense, spiral-poor concentrations such as Coma, to loose, spiral-rich systems like Pegasus; and there is no hint of any systematic dependence of the ABMHSC results on cluster type or $\mathrm{H} I$ deficiency. This is not really too surprising, since the cluster samples tend to be weighted by objects outside the central cores, and are therefore expected to be more akin to field galaxies in nature. A further indication that there is no dichotomy between the field and cluster samples is the fact that both independent sets of data yield the same value for Virgocentric motion. The details of Tammann's analysis are not available at the time of writing, so it is provisionally concluded that his lower expansion rate results from some linear combination of the second and third reasons mentioned above.

If there is a weakness in the ABMHSC value for $\mathrm{H}_{0}$, it surely rests with the fact that there are only three trustworthy calibrators at our disposal (whose Cepheid CCD photometry is still in a preliminary stage). When the number of reliable calibrators has grown into double digits, which will hopefully be the case a few years after the Hubble Space Telescope has commenced operation, we shall perhaps have a value for the expansion rate that is truly believable.

It is a great pleasure to thank the organizers of this Symposium for bringing us all to such a marvelous and stimulating country as China. Preparation of this article was partially supported with funds from United States National Science Foundation grant AST83-16629.

\section{REFERENCES}

Aaronson, M., Bothun, G., Mould, J., Huchra, J., Schommer, R. A., and Cornell, M. E.: 1986, Ap. J., 302, 536 (ABMHSC).

Aaronson, M., Huchra, J., Mould, J., Schechter, P. L., and Tully, R. B. : 1982, Ap. J., 258, 64 (AHMST).

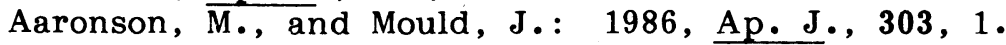

Balona, L. A., and Shobbrook, R. R.: 1984, M.N.R.A.S., 211, 375 .

Blanco, V. M., and Blanco, B. M.: 1985, Mem. Soc. Astron. Ital., 56, 15 .

Bothun, G.: 1986, private communication.

Burstein, D., Davies, R. L., Dressler, A., Faber, S. M., Lynden-Bell, D., Terlevich, R., and Wegner, G.: 1986, in "Galaxy Distances and Deviations from Universal Expansion," ed. B. F. Madore and R. B. Tully (NATO: Brussels); and private communication

(BDDFLTW).

Caldwell, J. A. R., and Coulson, I. M.: 1986, M.N.R.A.S., 218, 223. : 1987, in preparation.

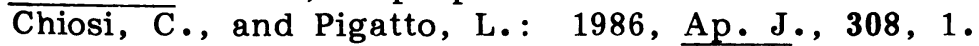

Christian, C. A., and Schommer, R. A.: 1986, preprint.

Collins, A., Joseph, R. D., and Robertson, N. A.: 1986, NATURE, 320, 506 . 
Conti, P. S., Garmany, C. D., and Massey, P. 1986, A. J., $92,48$. de Vaucouleurs, G.: 1980, Pub.A.S.P., 92, 579.

Eggen, O. J.: 1977, Ap. J. Suppl., 34, 1 .

Freedman, W. L.: 1985, in "IAU Colloqiuium 82, Cepheids: Theory and Observation," ed. B. F. Madore (Cambridge: Cambridge University Press), p. 225.

Frenk, C.S., and White, S. D. M.: 1982, M.N.R.A.S., 198, 173.

Graham, J. A.: 1984, in "Structure and Evolution of the Magellanic Clouds," ed. S. van den Bergh and K. S. de Boer (Dordrecht: Reidel), p. 207.

Kerr, F. J., and Lynden-Bell, D.: 1986, M.N.R.A.S., 221, 1023 .

Kraan-Korteweg, R. C., Cameron, L. M., and Tammann, G.: 1986, in "Galaxy Distances and Deviations from Universal Expansion," ed. B. F. Madore and R. B. Tully (NATO: Brussels).

Lilje, P. B., Yahil, A., and Jones, B. J. T.: 1986, Ap. J., 307, 91.

Lucey, J. R., Currie, M.J., and Dickens, R. J.: 1986, M.N.R.A.S., 221,453 .

Martin, W. L., Warren, P. R., and Feast, M. W.: 1979, M.N.R.A.S., 188, 139 .

Menzies, J. W., and Whitelock, P. A.: 1985, M.N.R.A.S., 212,783 .

Pritchet, C. J., and van den Bergh, S.: 1986, preprint.

Reid, I. N., and Strugnell, P. R.: 1986, M.N.R.A.S., 221, 887.

Reid, M. J., et al.: 1987, in "Star Formation," ed. M. Peimbert and and J. Jugaku (Dordrecht: Reidel).

Schmidt, E. G.: 1984, Ap. J., 285, 501.

Sandage, A.: 1986, private communication.

Sandage, A., and Carlson, G.: 1983, Ap. J. (Letters), 267 , L25.

Sandage, A., and Tammann, G. A.: 1984, NATURE, 307, 326.

Schommer, R. A.: 1986, in "Galaxy Distances and Deviations from Universal Expansion," ed. B. F. Madore and R. B. Tully (NATO: Brussels).

Schommer, R. A., Olszewski, E. W., and Aaronson, M.: 1984, Ap. J. (Letters), 285, L53.

Seidel, E., Da Costa, G. S., and Demarque, P.: 1986, preprint.

Vittorio, N., Juszkiewicz, R., and Davis, M.: 1986, NATURE, 323, 132.

Walker, A. R.: 1985, M.N.R.A.S., 212, 343 .

Yahil, A.: 1986, private communication. 


\section{DISCUSSION}

SILK: You used only three galaxies as distance calibrators for the infrared Tully-Fisher relation. If you instead used Tammann's calibration, revised to 1986 distance moduli as he just presented, what global value of $\mathrm{H}_{\mathrm{O}}$ would you obtain?

AARONSON: First, let me say that Tammann's "revised" moduli are his own, and not what I would necessarily agree with. I do not subscribe to the notion of blindly averaging together a wide variety of results. Second, by a judicious choice of calibrators I can give you an $\mathrm{H}$ anywhere between 65 and 100 . This is my whole point - the nearby distances are so poorly known, it is not too difficult to argue things up or down, which ever suits your bias.

ULMER: If you reduce distance to center of the Milky Way Galaxy, how does this affect the supernova rate/ brightness "problem" created by choosing $\mathrm{H}_{\mathrm{O}}=100$ ?

AARONSON: I believe it helps to alleviate this "problem." 